\title{
The Philani MOVIE study: a cluster- randomized controlled trial of a mobile video entertainment-education intervention to promote exclusive breastfeeding in South Africa
}

Maya Adam ${ }^{1,2,3^{*}}$ (D), Mark Tomlinson ${ }^{4}$, Ingrid Le Roux ${ }^{5}$, Amnesty E LeFevre ${ }^{6,7}$, Shannon A McMahon ${ }^{6,8}$, Jamie Johnston ${ }^{9}$, Angela Kirton ${ }^{2,3}$, Nokwanele Mbewu ${ }^{5}$, Stacy-Leigh Strydom ${ }^{5}$, Charles Prober ${ }^{1,2,3+}$ and Till Bärnighausen $8,10,11 \dagger$

\begin{abstract}
Background: In South Africa, rates of exclusive breastfeeding remain low and breastfeeding promotion is a national health priority. Mobile health and narrative entertainment-education are recognized strategies for health promotion. Inhome counseling by community health workers (CHWs) is a proven breastfeeding promotion strategy. This protocol outlines a cluster-randomized controlled trial with a nested mixed-methods evaluation of the MObile Video Intervention for Exclusive breastfeeding (MOVIE) program. The evaluation will quantify the causal effect of the MOVIE program and generate a detailed understanding of the context in which the intervention took place and the mechanisms through which it enacted change. Findings from the study will inform the anticipated scale-up of mobile video health interventions in South Africa and the wider sub-Saharan region.

Methods: We will conduct a stratified cluster-randomized controlled trial in urban communities of the Western Cape, to measure the effect of the MOVIE intervention on exclusive breastfeeding and other infant feeding practices. Eightyfour mentor-mothers (CHWs employed by the Philani Maternal Child Health and Nutrition Trust) will be randomized 1:1 into intervention and control arms, stratified by neighborhood type. Mentor-mothers in the control arm will provide standard of care $(\mathrm{SoC})$ perinatal in-home counseling. Mentor-mothers in the intervention arm will provide SoC plus the MOVIE intervention. At least 1008 pregnant participants will be enrolled in the study and mother-child pairs will be followed until 5 months post-delivery. The primary outcomes of the study are exclusive breastfeeding at 1 and 5 months of age. Secondary outcomes are other infant feeding practices and maternal knowledge. In order to capture human-centered underpinnings of the intervention, we will conduct interviews with stakeholders engaged in the intervention design. To contextualize quantitative findings and understand the mechanisms through which the intervention enacted change, end-line focus groups with mentor-mothers will be conducted.

(Continued on next page)
\end{abstract}

\footnotetext{
* Correspondence: madam@stanford.edu

Charles Prober and Till Bärnighausen are Co-Senior authors.

${ }^{1}$ The Department of Pediatrics, Stanford University, Stanford, CA, USA

${ }^{2}$ Stanford University's Digital Medical Education International Collaborative

(Digital MEdIC), Cape Town, South Africa

Full list of author information is available at the end of the article
}

C The Author(s). 2019 Open Access This article is distributed under the terms of the Creative Commons Attribution 4.0 International License (http://creativecommons.org/licenses/by/4.0/), which permits unrestricted use, distribution, and reproduction in any medium, provided you give appropriate credit to the original author(s) and the source, provide a link to the Creative Commons license, and indicate if changes were made. The Creative Commons Public Domain Dedication waiver (http://creativecommons.org/publicdomain/zero/1.0/) applies to the data made available in this article, unless otherwise stated. 
(Continued from previous page)

Discussion: This trial will be among the first to explore a video-based, entertainment-education intervention delivered by CHWs and created using a community-based, human-centered design approach. As such, it could inform health policy, with regards to both the routine adoption of this intervention and, more broadly, the development of other entertainment-education interventions for health promotion in under-resourced settings.

Trial Registration: The study and its outcomes were registered at clinicaltrials.gov (\#NCT03688217) on September 27th, 2018.

Keywords: Mobile health, Video, Narrative, Entertainment-education, Community-based, Community health worker, Humancentered design, Maternal child health behavior, Breastfeeding, South Africa

\section{Background}

Despite global progress in reducing maternal, newborn and child mortality, more than 5 million children, around the world, die before reaching their fifth birthday [1]. Rates of under-5 mortality are highest in subSaharan Africa and, in 2015, 45\% of these deaths occurred in newborns. A majority of under-5 deaths are the result of preventable diseases, the incidence of which could be reduced by implementing health-promoting maternal-child behaviors, such as exclusive breastfeeding (EBF) [1-4].

The health benefits of EBF, especially in low- and middle-income countries (LMIC), have been well documented [5]. For the child, these include protection against infectious diseases, improved measures of intelligence and decreased risk of becoming overweight and developing diabetes. EBF also confers health benefits for the mother, including a reduced risk of breast and ovarian cancer [5]. Globally, EBF could save an estimated 823,000 under-5 lives each year, as well as preventing 20,000 maternal deaths from breast cancer. Yet, despite World Health Organization recommendations that children should be exclusively breastfed for the first 6 months of life, EBF rates hover at only 37\% in LMIC [5].

South Africa has one of the lowest rates of EBF in the world [5]. Estimates of EBF rates under- 6 months in South Africa range from $8 \%[5,6]$ to the most generous estimate, 32\% [7]. Furthermore, the percentage of children exclusively breastfed appears to decrease sharply over the first 6 months, from $44 \%$ of infants aged $0-1$ month to $24 \%$ of infants aged 4-5 months [7]. The health problems that result are largely due to unsafe formula feeding and the widespread practice of introducing solid foods early (often before 3 months) into the infant's diet [8]. In South Africa, some reports suggest that more than $70 \%$ of infants are given solid foods before reaching 6 months, the recommended age for initiating complementary feeding [8]. Unsafe formula feeding, including the use of bottles with poorly cleaned nipples, can lead to diarrheal disease [9] from pathogens entering the gut. Solid foods, introduced too early into a child's diet, can also cause gastro-intestinal infections as well as nutrient deficiencies when these foods displace breastmilk without providing adequate nutrient density [10]. The limited availability of reliable national data on infant feeding in South Africa complicates the design and evaluation of infant feeding interventions [8]. However, systematic reviews of the literature suggest that large-scale interventions focused on educating mothers can increase the prevalence of EBF and decrease infant mortality [11-14].

The narrative entertainment-education (E-E) approach to health education appears to be a promising strategy for promoting health behavior change $[15,16]$. E-E content endeavors to deliver health messaging through an entertainment media framework. A growing body of scientific evidence suggests that $E-E$ is an effective approach towards positively influencing beliefs, attitudes and behaviors [15, 17-20]. Especially in populations with low motivation and/or a reduced ability for cognitively evaluating the intended health messaging, E-E may be a powerful health education strategy [15]. The characteristics of effective E-E include: a) appealing narratives, b) high production quality, c) persuasive messages that are unobtrusive and d) high potential for involvement or identification with the presenters or characters portrayed [15]. While E-E can take many forms, a video-based approach readily supports the harmonious integration of these characteristics.

Video-based content, optimized for mobile devices, may also facilitate broad dissemination of health education, especially as the global penetration of mobile technology increases. Recent advances in mobile messaging have already begun to facilitate the delivery of health messages at scale [21-23] and researchers are increasingly recognizing the potential for mobile phones and tablets to play an important role in health education interventions in LMIC [23-26]. South Africa is an example of a LMIC at the forefront of mobile health (mHealth) initiatives, due to its rapidly expanding penetration of mobile phones and national internet infrastructure [27-29]. National, maternal-child mHealth initiatives built around free, text-based SMS messaging have attracted global attention and been well received [30, 31]. General "tech-savviness" in South Africa continues to grow, with 
$37 \%$ of adults reporting smartphone ownership and $42 \%$ reporting daily internet usage in 2016 [32]. Active experimentation with WhatsApp, a widely used mobile communication tool that supports the transfer of videos, is also underway in many sectors, including health [33, 34].

Even for South Africans who do not yet have access to mobile phones, a feasible dissemination pathway lies in the delivery of educational videos by community health workers (CHWs) who bring teaching tablets to their in-home counseling sessions [35]. Interventions that support women in their homes have demonstrated efficacy in improving breastfeeding rates [36]. The Philani Maternal Child Health and Nutrition Trust [37] is an example of a successful community based organization, employing "mentor-mothers", CHWs who offer in-home health promotion counseling to pregnant women and mothers within their neighborhoods. Data from both case studies and quantitative research suggest additional improvements in breastfeeding rates when synergistic interventions - for example, mass media interventions are used to supplement successful community-based programs [36]. Developing interventions that boost the efficacy of community-based programs may be a critical step towards ending preventable child and maternal deaths by 2030 (as called for by the World Health Organization and UNICEF) [38]. Prior research in South Africa suggests that such synergistic interventions are even more effective when they are created in close collaboration with community-based programs, actively involving local stakeholders in the content creation process [39].

Applying a human-centered design (HCD) approach to the development of health education interventions may be a powerful way of tailoring these interventions to the specific settings and needs of their target communities, a priority identified in the 2016 Lancet Series on Breastfeeding [36]. The HCD approach has been described as "constructive, experiential and rooted in the needs and context of end-users of a product or service" [40], with the end goal of developing novel solutions to pressing problems. The creation of health education using an HCD approach involves several principles and practices that have been well characterized in the literature [41]. These include focusing on an empathic understanding of the target audience, creating content through a process of rapid prototyping, gathering of feedback and responsive iteration. Finally, the HDC approach is characterized by a relatively high tolerance for ambiguity and failure during the design process [40, 42].

Recent systematic reviews of mHealth interventions for improving maternal and neonatal health outcomes have called for: 1) strong experimental research designs including randomized controlled trials, 2) feasibility research, 3) government involvement and 4) integration of mHealth interventions into the healthcare system [23]. The Philani MOVIE intervention will be evaluated using a randomized-controlled trial, which follows a successful, qualitative feasibility study [35] involving the same population of CHWs who will be delivering the intervention in this trial. The 13-video Philani MOVIE intervention was created in collaboration with the Western Cape Department of Health, the South African National Department of Health, the Philani Maternal Child Health and Nutrition Trust and UNICEF, among other local stakeholders. By integrating the Philani MOVIE intervention into the successful, community-based Philani Mentor Mother Outreach Program [37], we aim to explore the potential for innovative, mobile video interventions to positively impact breastfeeding rates in South Africa, an important predictor of maternal and neonatal health outcomes.

\section{Aims}

This study will achieve the following specific research aims: To

1. Establish the

a. Effectiveness of the Philani MOVIE intervention for increasing the practice of exclusive breastfeeding

b. Effectiveness of the Philani MOVIE intervention for improving other infant feeding practices and maternal knowledge about infant feeding

2. Determine the usefulness of human-centered design principles when applied to the development of mobile health interventions

3. Elucidate the mechanisms of intervention action and the acceptability of the intervention to CHWs.

\section{Methods}

\section{Study setting and implementation partner}

This study will be conducted within the under-resourced settlements of the Western Cape Province in South Africa. In 2010, the infant mortality rate in the Western Cape was reported to be 25 per 1000 live births [43]. A 2014 study of under-resourced communities in the Western Cape, found the EBF rate to be only 6\% [44]. Operating within these communities (see Fig. 1), the Philani Maternal Child Health and Nutrition Trust [37] is a non-government organization serving approximately 100 under-resourced neighbourhoods. The Philani Mentor Mother Outreach Program is focused on improving child health outcomes through the deployment of "mentor-mother" CHWs. Mentor-mothers are identified as positive role models within their communities, after they have demonstrated success in raising healthy families despite resource shortages. Once recruited, mentor-mothers complete a standardized training program for delivering 


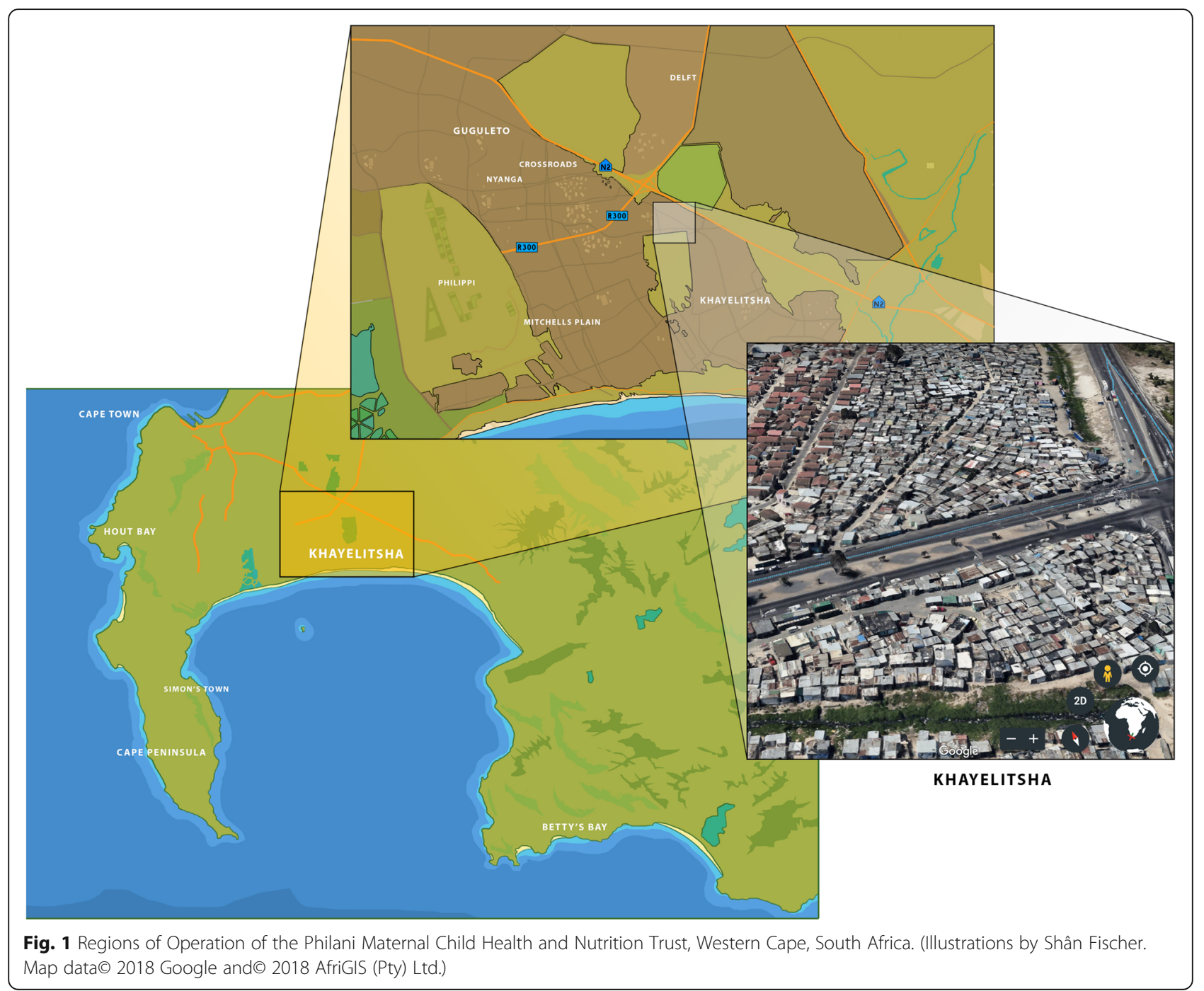

in-home health promotion counselling services within their communities. Among other health-promotion tasks, Philani mentor-mothers are trained to monitor growth, counsel parents around proper nutrition and refer sick family members to the nearest clinic when necessary. These mentor-mothers promote a variety of health behaviours, including those related to HIV-prevention, breastfeeding, child nutrition, growth and development $[37,45,46]$.

\section{Description of the intervention}

The Philani MOVIE intervention consists of 13 short, (2-5 min.) educational videos created over the course of 10 months in collaboration with community members and local stakeholders in maternal-child health. The health messaging in each video is closely aligned with the most recent WHO recommendations for infant feeding and narrated in English and isiXhosa, the languages spoken most widely among the study participants. The videos use simple syntax, avoiding medical jargon. Table 1 summarizes the video topics included in the Philani MOVIE intervention.

\section{Intervention development}

A human-centered design approach was applied during the development of the intervention. Formative feedback

Table 1 Philani MOVIE video topics

1. Trailer

2. A Breastfeeding Story

3. The Benefits of Breastfeeding

4. How Breastfeeding Works

5. Breastfeeding Recommendations

6. A Kangaroo Mother Care Story

7. Common Challenges Faced by Breastfeeding Mothers

8. What Happened to the Practice of Breastfeeding?

9. Unsafe Infant Feeding Practices

10. Breastfeeding and HIV

11. Tips for Working Mothers who Breastfeed

12. When Breastfeeding Isn't Possible

13. A Stunting Prevention Story 
was solicited from end-users within the target audience as well as experts in multiple disciplines, including community medicine, academia, public health, media marketing and the performing arts. Scripts were collaboratively edited by content experts, who worked in parallel using Google Drive to allow for reliable version control. Early drafts of videos were then shared via WhatsApp with community members, most of whom did not use email regularly, informing a process of Rapid Iterative Testing and Evaluation [47, 48]. Feedback from community members was gathered via WhatsApp and during face-to-face "bodystorming" (physical brainstorming) sessions $[47,49]$ in which mentor-mothers tested the intervention through role-play activities involving mockcounseling sessions. Feedback was synthesized on a rolling basis, informing multiple iterations of the content. In this way, the health messages, language, illustrations, characters, color palate, narrative delivery style, logo and soundtrack were collaboratively and iteratively finalized over the 10 -month content creation period. Key health and motivational messages were interwoven with animations created by local artists and underscored by the narratives of community mothers and South African celebrity mothers. Both community members and media marketing experts emphasized the importance of representing and celebrating South Africa's diverse ethnic profile. This feedback shaped production decisions about both the illustrations included in the intervention and the profiles of the mothers featured in the intervention video footage. The trailer for the series can be previewed here https:// youtu.be/1sss8ViPKJo [50].

\section{Trial design}

Our study is a cluster-randomized controlled trial with baseline covariate adjustment and stratification [51] (See Fig. 1). The randomization unit is the mentor-mother. Each mentor-mother will enroll several pregnant women over the course of the trial. We conducted analyses of routine program data from these mentor-mothers and found that, among a range of available covariates, the only significant predictor of EBF was the type of neighborhood in which the mentor-mother lived and performed her health promotion duties. These neighborhood types include: informal, formal and mixed neighborhoods. We thus decided to stratify the randomization according to neighborhood type, to ensure balance of intervention versus control assignment by this covariate. Based on the existing literature [11, 52-59], we also expected that the following covariates would be associated with the primary outcome measure:

1. participant's number of previous children

2. participant's age

3. running water in the home
4. electricity in the home

5. participant's employment status

6. participant's education level

To further increase the statistical efficiency of our analysis, we thus decided to measure these covariates at baseline and adjust for them in our primary analyses, in addition to baseline neighborhood type. Randomization of the mentor-mothers was performed, using a computer-generated random allocation sequence, by faculty at Heidelberg University, Germany. The allocations were then transferred by email to Philani for implementation. Cluster randomization was preferred over individual randomization in this trial because each mentor-mother is responsible for counseling pregnant women within her neighborhood, making individual participant randomization logistically impossible without disrupting existing protocols.

\section{Sample size calculation}

Our sample size calculation was based on the primary outcomes: EBF at 1 and 5 months of age. We performed the calculations using standard methods for cluster-randomized controlled trials with baseline covariate adjustment and stratification [60]. The randomization units were the mentor-mothers. We assumed an intra-cluster correlation of our two primary outcomes of 0.1 [61]. We used routine program data describing the performance of the 84 mentor-mothers who will participate in this trial to inform our power calculation. Based on this data, and a range of other data sources on breastfeeding in South Africa [6-8, 36, 57, 62, 63], we assumed that $40 \%$ of mothers exclusively breastfed their infants at 1 month of age and $10 \%$ of mothers exclusively breastfed their infants at 5 months of age. Assuming a correlation between the baseline measurements and the primary outcome of 0.30 and an average enrolment of 12 pregnant women per mentor-mother over the course of this study, we estimated that at the $5 \%$ significance level the trial would have $80 \%$ power to detect a 13 -percentage point increase in EBF at 1 months of age and a 9-percentage point increase in EBF at 5 months of age. This minimal detectable difference met our condition for policy relevance (an improvement of more than 15 percentage points). We thus set the total sample size for outcome assessment at 840 pregnant women plus 20\% (to allow for loss to follow-up), i.e. 1008 pregnant women. Figure 2 illustrates the trial design.

\section{Trial outcome measures}

The outcome measures in this trial are based on the most recently published WHO indicators for the study of feeding practices in infants [64] and the most recent national infant feeding data for South Africa [7]. 

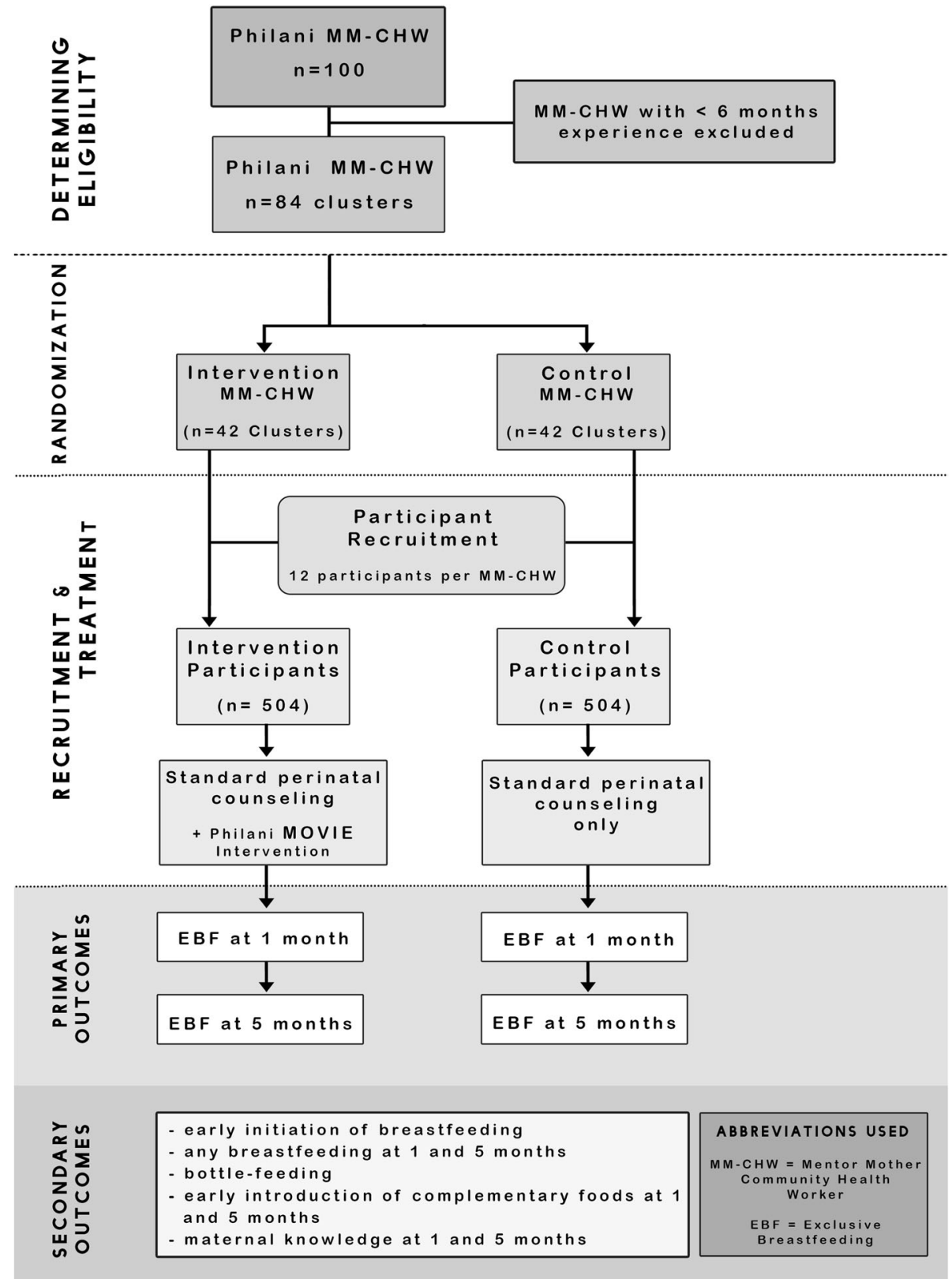

Fig. 2 Trial Design for the Philani MObile Video Intervention for Exclusive breastfeeding (MOVIE) Study

The primary outcomes will be short-term EBF (at 1 month) and long-term EBF (at 5 months). Primary outcomes will be measured using both point-in-time (24-h recall) and life-long (since birth) data, as recommended in the literature [65]. Primary outcomes data will be collected via tablet-based questionnaires and verified by follow-up telephone surveys, administered within one week of each tablet-based survey.

Secondary outcomes will include a) early initiation of breastfeeding $(<1 \mathrm{~h}$ after delivery, measured by recall on tablet-based survey at 1 month and verified by follow-up phone survey), b) any breastfeeding at 1 and 5 months (based on 24-h recall on tablet-based survey and verified by follow-up phone survey), c) bottle-feeding (based on 24-h recall on tablet-based survey and verified by follow-up phone survey), early introduction of complementary foods at 1 and 5 months (based on 24-h and since-birth recall, measured by phone surveys) and maternal knowledge at 1 and 5 months (measured by phone surveys).

Table 2 summarizes the quantitative outcomes that will be examined in this trial. 
Table 2 Outcomes for the Philani MOVIE trial

\begin{tabular}{ll}
\hline Outcome Label & Outcome Definition \\
\hline A. Primary outcome & \\
$\begin{array}{l}\text { 1. Short-term exclusive } \\
\text { breastfeeding (24-h recall) }\end{array}$ & $\begin{array}{l}\text { Infant, age } 1 \text { month, was exclusive } \\
\text { breastfed in the past } 24 \mathrm{~h}\end{array}$ \\
$\begin{array}{l}\text { 2. Long-term exclusive } \\
\text { breastfeeding (24-h recall) }\end{array}$ & $\begin{array}{l}\text { Infant, age } 5 \text { months, was exclusiv } \\
\text { breastfed in the past } 24 \mathrm{~h}\end{array}$ \\
B. Secondary outcomes & \\
$\begin{array}{l}\text { 1. Short-term exclusive } \\
\text { breastfeeding (since birth recall) }\end{array}$ & $\begin{array}{l}\text { Infant, age } 1 \text { month, has been } \\
\text { exclusively breastfed since birth }\end{array}$ \\
$\begin{array}{l}\text { 2. Long-term exclusive } \\
\text { breastfeeding (since birth } \\
\text { recall) }\end{array}$ & Infant, age 5 months, has been \\
\end{tabular}

\author{
3. Early initiation \\ of breastfeeding \\ 4. Any breastfeeding \\ at 1 month \\ 5. Any breastfeeding \\ at 5 months \\ 6. Bottle-feeding
}

7. Early introduction of complementary foods at 1 month (24-h recall)

8. Early introduction of complementary foods at 5 months (24-h recall)

9. Early introduction of complementary foods at 1 month (since birth recall)

10. Early introduction of complementary foods at 5 months (since birth recall)

11. Maternal knowledge at 1 month post-delivery

12. Maternal knowledge at 5 months post-delivery
Infant was breastfed within the first hour of life (based on recall at 1 month)

Infant, age 1 month, received any breastmilk in the past $24 \mathrm{~h}$, even if not exclusively breastfed

Infant, age 5 months, received any breastmilk in the past $24 \mathrm{~h}$, even if not exclusively breastfed

Infant, under 6 months of age, was fed using a bottle with a nipple in the past $24 \mathrm{~h}$.

Infant, age 1 month, has received complementary foods in the past $24 \mathrm{~h}$.

Infant, age 5 months, has received complementary foods in the past $24 \mathrm{~h}$.

Infant, age 1 month, has received complementary foods at some point since birth.

Infant, age 5 months, has received complementary foods at some point since birth.

Maternal knowledge of breastfeeding current recommendations and basic health principles relevant to infant feeding measured at 1 month post-delivery.

Maternal knowledge of breastfeeding current recommendations and basic health principles relevant to infant feeding measured at 5 months post-delivery.
Method of Measurement

These outcomes will be measured by tabletbased surveys, adapted from the South Africa Demographic and Health Survey 2016 [7] and following the most recent WHO indicators for infant feeding practices [64]. A point-in-time or current status measurement (based on 24-h recall) is presently the most common method for measuring exclusive breastfeeding [65]. Responses to initial tablet-based surveys administered at 1 and 5 months will be verified by follow-up phone surveys, conducted by an independent, vetted phone survey research company, within one week of the initial data collection timepoint. Life-long (since birth recall) data on exclusive breastfeeding, will also be collected and verified by phone survey. Reporting indicators based on both point-in-time and life-long data has been recommended as a stronger approach than either of these in isolation [65].

This outcome will be measured in the 1 month tabletbased survey and verified by phone survey within one week of the initial data collection timepoint.

These outcomes will be measured by tablet-based surveys at 1 month and 5 months, adapted from the South Africa Demographic and Health Survey 2016 [7] and following the most recent $\mathrm{WHO}$ indicators for infant feeding practices [64]. Responses to tablet-based surveys will be verified by follow-up phone surveys, conducted by an independent, vetted phone survey research company, within one week of the initial data collection timepoint.

These out comes will be measured by phone surveys at 1 and 5 months. Participants will respond to an infant feeding questionnaire, incorporating both point-in-time and life-long data, adapted from previously published infant feeding measurement tools [82-85] and informed by the WHO indicators for infant feeding practices [64].

These outcomes will be measured by phone survey questionnaires on maternal knowledge about breastfeeding (developed by study team, adapted from previously published breastfeeding knowledge assessment tools $[59,86,87])$.

\section{Qualitative component}

Two qualitative components will be nested within this $\mathrm{RCT}$ in order to capture the human-centered design process of the intervention development, contextualize the results of the trial and understand the mechanisms through which the MOVIE enacted change. The qualitative methods used in this study will include in-depth interviews (IDIs) and focus group discussions (FGDs). Sampling for both qualitative study components will be purposive [66] and, to the extent possible given financial and logistical constraints, qualitative data collection will continue until saturation and redundancy are reached $[67,68]$. All qualitative research assistants will work under the direct supervision of the research team and will be trained on qualitative methods. Data will be collected in a language of the respondents choosing and following informed consent. As soon as possible following the conclusion of a qualitative data collection activity, debriefings between qualitative research assistants and the study lead(s) will occur [69]. Debriefings will 
allow the study lead(s) to gain rapid insights into the content of data and strengthen not only the skills of data collectors but also the quality and trustworthiness of data in real-time [69]. All qualitative data will be tape-recorded, transcribed, translated into English as necessary, and quality checked for consistency and accuracy.

\section{In-depth interviews on human-centered approaches in Mobile video design}

IDIs will be conducted with stakeholders engaged in the design of the MOVIE intervention from concept generation to early prototyping to final refinement. Stakeholders will include those whose insights effectively shaped the content and delivery of the intervention including: Department of Health officials, representatives from governmental, non-governmental and UN agencies, health staff employed by Philani, film producers, graphic designers, marketing specialists, donors and academic faculty experts. Sampling for IDIs will be done via the snowball method wherein the study team will approach the Department of Health and key programmatic personnel engaged in the conception and development of the MOVIE intervention videos. These individuals will assist the research team in identifying initial respondents. These initial respondents will then be asked to assist in the identification of other respondents, who might facilitate an understanding of how and why the intervention was modified and refined in the course of video development.

\section{Focus group discussions to contextualize and interpret quantitative findings}

FGDs will be conducted with mentor-mothers following analysis of the quantitative data as a means to "explain" and thus to better interpret the quantitative results [70]. FGDs will serve as an opportunity to explore the heterogeneity in effects we expect to observe across mentor-mothers and the communities they serve, and to capture information about contextual elements that foster or hinder change. This information may prove particularly informative in the context of a later national rollout, wherein knowledge about the conditions and characteristics necessary to produce change will be actively sought. The type of purposive sampling to be employed will be criterion sampling wherein characteristics that emerge as relevant based on the quantitative results (such as mentor-mother age, place of residence or years of service) will shape the nature of the sample for the qualitative study [66].

\section{Data collection and pre-trial training}

Mentor-mothers in both the intervention and control groups will undergo training on how to obtain written, informed consent, record baseline variables and collect data on infant feeding practices directly on their tablets. This data will be collected as close as possible to the 1 month and 5 months post-delivery timepoints, $(+/-2$ weeks) via infant feeding questionnaires. These questionnaires have been translated into isiXhosa and audio-recorded to help overcome literacy barriers. They will be filled out directly on the tablets by the participants. To standardize the collection of data, all 84 mentor-mothers will carry tablets with the infant feeding questionnaires for the duration of the study period. Only half [42] of the tablets will be loaded with the Philani MOVIE video intervention. All mentor-mothers will be trained on how to charge, use, troubleshoot and care for their tablets. These tablets are 8" Android devices with 16GB of storage. The videos can be played offline and questionnaires completed and stored on the tablets while a mentor-mother is conducting home-visits in areas without internet access. Once the mentor-mother re-connects to the internet, all completed questionnaires will automatically be forwarded to the local research staff, based in Cape Town, for de-identification, cleaning and analysis.

Each tablet-based survey will be followed, within one week, by a phone-based survey conducted by Social Surveys, a professional telephone research company, located in South Africa. The use of telephone surveys for verification and follow-up after initial face-to-face interviews has demonstrated significant benefits. A dual sampling frame approach (using a combination of face-to-face and telephone interviews) has been recommended for such surveys, particularly for low-income and educationally under-resourced respondents [71-73]. Computer-assisted telephone interviewing (CATI) will also be employed to enhance accuracy of data collection and reporting. All data will be de-identified by local research staff in South Africa and carefully stored in the locked, secure offices of the local research team. Only de-identified data will be shared with collaborating research partner institutions for data analysis purposes.

Mentor-mothers in the intervention group will receive additional training on how to deliver the Philani MOVIE videos, which are designed in a modular fashion such that they can be viewed in any order, and thus tailored to the needs of the participant. Video sequencing decisions will be made by the mentor-mothers, who are trained by Philani to facilitate close alignment between health messages delivered during home-visits and the individual needs of each participant at each home visit and perinatal stage. Mentor-mothers in the intervention group will be instructed to administer each of the 13 intervention videos at least once to each participant during the study period. Video views will be tracked directly on the tablets and recorded on a paper-based tracking form by the mentor-mothers. The Philani MOVIE intervention will be delivered in parallel to the Standard 
Philani Perinatal Counseling Program (SoC), which includes counseling on infant feeding (see Table 3).

\section{Trial participants and recruitment}

Participants in the trial will be 84 clusters of consenting, adult, pregnant clients (at least 1008 participants aged 18 years or more), living within the neighborhoods of 84 resident mentor-mothers and enrolled in the Philani Mentor Mother Outreach Program. Philani mentormothers are assigned to work only in the neighborhoods in which they live, resulting in 84 clusters of participants. Mentor-mothers with less than 6 months of experience and participants under 18 years of age will be excluded from the study. Based on Philani estimates, each mentor-mother typically identifies and enrolls 2-3 pregnant women per month within her neighborhood. Study participants will be enrolled on an ongoing basis until 1008 participants are enrolled. Written, informed consent will be collected from all study participants by a trained mentor-mother, prior to the collection of any data. Participants will be made aware that they have the right to withdraw from the study at any time. The study will conclude when the babies born to the enrolled participants during the study period have reached 5 months of age. Eligible pregnant participants will be recruited after their 20th week of pregnancy.

\section{End-line focus group participants}

End-line focus group participants will be the 42 mentormothers randomized to the intervention arm of the study. These mentor-mothers will be invited to take part in this explanatory, qualitative portion of the study after they have gained experience administering the Philani MOVIE intervention for 12 months.

\section{In-depth interview participants}

IDI participants will be consenting participants from the group of content experts, local stakeholders and community members who participated in the Philani MOVIE content creation process. Interviews will be conducted with experts in community medicine, academia, public

Table 3 Topics Addressed in Standard Philani Infant Feeding Counseling Sessions

1. Maternal beliefs about feeding

2. The practice of exclusive breastfeeding

3. The benefits of breastfeeding and the composition of breast milk

4. How milk is produced and released from the breast

5. How to position and attach a baby for breastfeeding

6. How to express breast milk

7. Common feeding difficulties and breast conditions relating to breastfeeding

8. Infant feeding for HIV positive mothers

9. Safe infant feeding for women who meet have chosen not to breastfeed and meet the AFASS criteria for formula feeding (Acceptable, Feasible, Affordable, Sustainable and Safe) health, media marketing and the performing arts, as well as the community mothers who helped to create the intervention and are representatives of its target audience.

\section{Data analysis \\ Quantitative analysis of the trial results}

Our primary analysis will be based on intention-to-treat (ITT) at the unit of the individual newborn/individual mother, with standard errors adjusted for clustering at the level of the unit of randomization, i.e., the mentormothers. We will adjust for baseline neighborhood type in addition to the six additional baseline covariates described above (participant's number of previous children, participant's age, running water in the home, electricity in the home, participant's employment status, and participant's education level).

We will use mixed-effects generalized linear models for our primary analysis, using a fixed effect for the assignment to treatment vs. control arm and a random effect for mentor-mother. In particular, we will use modified Poisson models (generalized linear models with Poisson distribution and a logarithmic link function), for our binary outcomes, i.e., the primary outcome, as well as secondary outcomes 1-6 (see Table 1). We chose modified Poisson models because they generate estimates of risk ratios (and thus avoid the well-known interpretational difficulties associated with odds ratios) and because they converge more easily than alternative models that generate risk ratios (such as binomial models) [74]. For the continuous secondary outcomes (outcomes 11 and 12 - see Table 1), we will use generalized linear models with Gaussian distribution and identity link function.

\section{Qualitative analysis}

Debriefings following the conclusion of a qualitative data collection activity will corroborate and refine a template for thematic analysis [69]. In line with an underlying Grounded Theory approach, analysis will begin with a first reading of debriefing notes and transcripts to acquire familiarity with the data by the qualitative research team [75]. Categories and sub-categories will be developed, modified and expanded upon based on themes that emerge as analysis proceeds. An initial phase of open, inductive coding on a selection of rich, diverse and representative transcripts will be conducted. As new themes and theories emerge in the data, the research team will test theoretical notions and revisit earlier transcripts and debriefing notes in order to build a theory [75]. This analysis will be supported with software such as NVivo [76, 77]. In line with the principles of Grounded Theory, an extended literature review will follow the completion of coding [75]. 


\section{Theory of change}

This work rests upon the theoretical underpinnings of the Elaboration Likelihood Model (ELM) [78], which suggests that there are two contributing pathways to achieving the changes in attitude that predict a desired behavioral outcome such as EBF. The first "central route" is influenced by the learner's motivation and ability to cognitively process the information presented (ie: the health benefits of EBF). This can be influenced by factors such as the length of the content and the degree to which the language used is understood by the learner. The second "peripheral route" relies on cues embedded in the method of information delivery that contribute to its relative acceptability to the learner. Positive, peripheral cues, such as the learner's subjective evaluation of the person delivering the information or the learner's emotional involvement in the content, lead to peripheral attitude changes which, while less enduring than central attitude changes, can positively influence the learner's motivation to process the messaging via the central route
[78]. Both ELM and a variation on this model, called the extended Elaboration Likelihood Model (eELM) have been used to explain the efficacy of entertainment-education in changing leaner attitudes and behaviors in response to health messages and other types of persuasive messages [16, 19, 79]. According to the eELM model, effectively designed messages, containing positive peripheral cues, can reduce resistance to attitude changes (counter-arguing), when the learner is "transported" (engaged) in the narrative or identifies with its characters. This identification can be empathic or cognitive, through perceived similarity or wishful identification (a desire to emulate the characters featured). This model even suggests that the imagined "pseudo relationships" than can develop between viewers in the general public and on-screen celebrities, can be harnessed as a powerful peripheral cue to enhance attitude change in persuasive messaging [79]. Fig. 3 illustrates the intersecting theories of change on which our intervention is grounded.

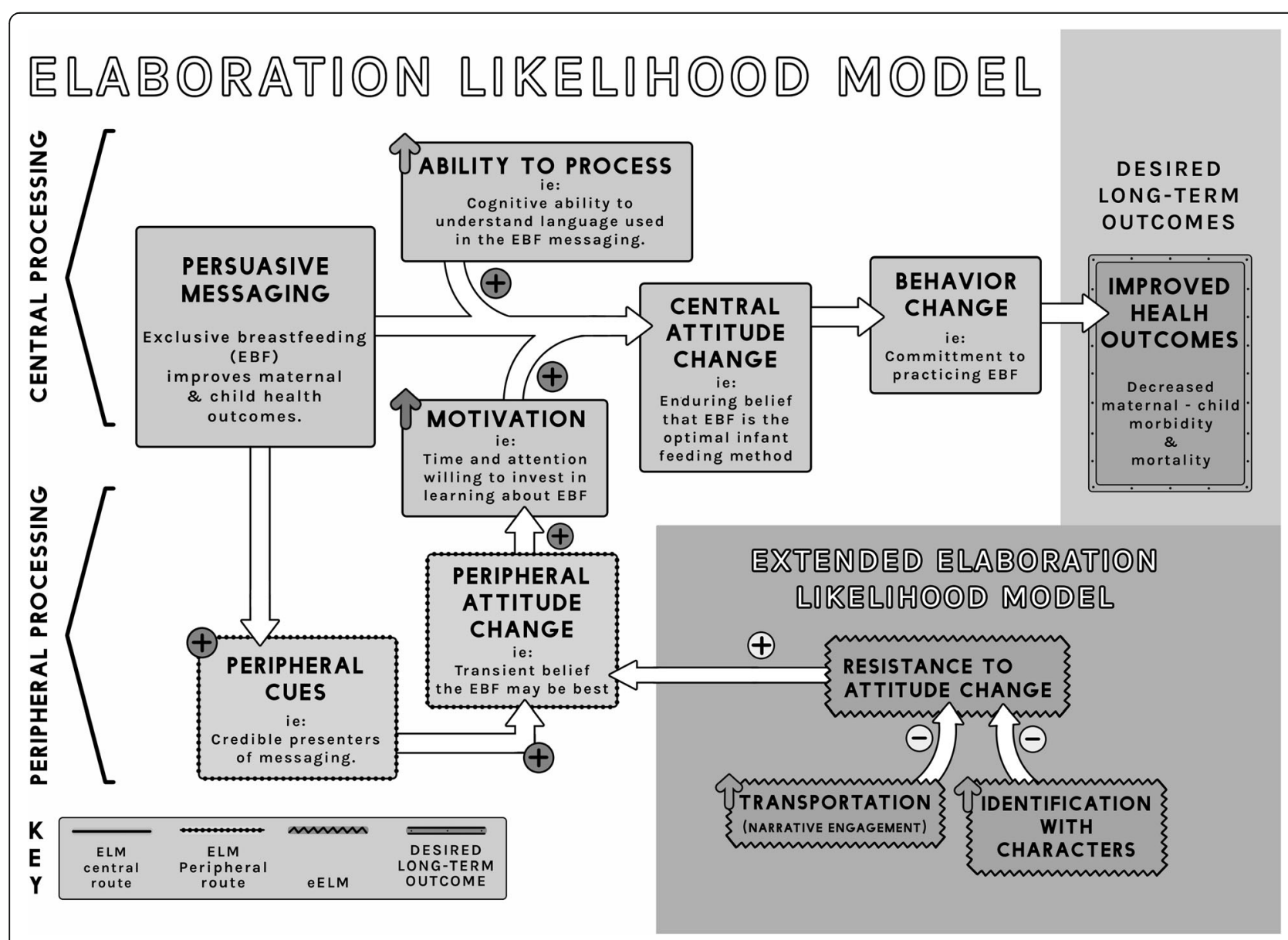

Fig. 3 Intersection of the Elaboration Likelihood Model (ELM) and the extended ELM (eELM) theoretical models with desired long-term health outcomes 


\section{Study governance and oversight}

This study will benefit from oversight by a data and safety monitoring board (DSMB), which will consist of a senior biostatistician, a senior local health systems researcher and a professor of pediatrics and health policy with expertise in primary care and health outcomes research. The DSMB will meet twice per year. It will evaluate the study progress and provide guidance on study continuation and any potential changes in study conduct in the case of unexpected events or operational difficulties.

\section{Discussion}

The trial described in this paper aims to measure the causal effect of a mobile, video-based entertainment-education intervention, developed using human-centered design principles, on infant feeding behaviors in underresourced South African communities. Nested qualitative components, involving interviews with local stakeholders and FGDs with mentor-mothers, will explore the development of the intervention and contextualize the results of the trial, while helping to characterize the mechanisms through which the Philani MOVIE intervention enact change.

The application of HCD principles is a new and promising complementary approach to the design of global health interventions [40]. A key principle of HCD is the idea that content should be created with the needs and context of the target community in mind. The intervention in this trial is based on a strong theory of change and was developed as part of a human-centered design study. Multiple cycles of formative feedback and rapid iteration generated an intervention that is intended to be rooted in the needs and context of the communities it addresses. Creating educational videos that are primarily visual, with carefully scripted audio tracks that convey health messages simply, can further align such content with the needs and context of its target community, especially in low-literacy settings. Additionally, translation of the content (into all 11 South African national languages) could further increase the scalability of the intervention and is underway. The organization of the intervention into short, modular videos was intended to optimize engagement and facilitate their flexible use and dissemination via multiple mobile technology pathways.

Applying the principles of community-based research $[80,81]$ to the design and production of this intervention facilitated the HCD approach to its development. We harnessed the strengths and resources within the target communities by soliciting their feedback on early prototypes and their active participation in the content creation process. The resulting intervention includes the stories and voices of local community mothers, presented alongside the parallel narratives of local celebrities. The decision to represent a variety of ethnicities, was made in an attempt to create an intervention that would resonate broadly across multiple South African demographics. The idea of including celebrities' stories originated within the community and was implemented with the goal of emphasizing the common aspirations and challenges faced by many new mothers. These narrative elements were also included as positive peripheral cues, to optimize peripheral processing of health messages through realistic identification with community mothers and wishful identification with celebrity mothers. As a result, the intervention aims to inhibit resistance to attitude change (counter-arguing) as posited by the theoretical models underpinning this intervention. By partnering closely with target communities and local stakeholders during the intervention development, we were able to: a) identify and build on the strengths and resources within our target communities, b) collaboratively define priority problems and the desired characteristics of the intervention intended to address them and c) use an iterative-feedback process, with a high tolerance for failure and rapid reiteration, to create an intervention that is closely tailored to the needs and context of the target audience.

In the Philani MOVIE intervention, mobile and other emerging technologies facilitate the dissemination, but also the iterative HCD development of the content. The use of Google Drive to collaboratively edit scripts in parallel with version control and the ability to share drafts of video content with community members by WhatsApp facilitated a brisk production timeline for the intervention. The use of WhatsApp and other messaging tools to solicit rapid feedback at key decision points, even from community collaborators who were uncomfortable using email, may eventually prove to be a powerful addition to the production workflow for community-based health education content.

In addition to facilitating the content creation process, the growing penetration of smartphones, tablet technology and general tech-savviness in LMIC yield promising pathways for disseminating content to under-resourced communities. This dissemination can be a) mediated by community health workers or b) direct-to-learner approaches in future initiatives where every family has access to a smartphone or tablet. In anticipation of these emerging pathways, we find ourselves compelled to create a comprehensive, multi-lingual, free, accessible, engaging and impactful "video library for health". Such a library would allow for timely updating, ongoing iterative improvement and broad dissemination of critical, preventive health education messages.

To this end, our research is intended to support capacity building for a "next generation" of digital, 
maternal-child health education, a generation of innovative educational tools, rooted in the needs and contexts of the audiences they intend to serve. By doing so, we believe we have an opportunity to support the adoption of behaviors that will form the cornerstone of healthier and more prosperous futures for mothers and children around the world. This cluster-randomized controlled trial serves to provide rigorous scientific evidence about the causal effectiveness of one particular intervention in this broader workstream.

\section{Abbreviations}

CATI: Computer-assisted telephone interview; CHW: Community health worker; DSMB: Data and safety monitoring board; EBF: Exclusive breastfeeding; E-E: Entertainment education; eELM: Extended elaboration likelihood model; ELM: Elaboration likelihood model; FGD: Focus group discussion; HCD: Human-centered design; IDI: In-depth interview; LMIC: Lowand middle-income countries; MM-CHW: Mentor-mother community health worker; MOVIE: Mobile video intervention for exclusive breastfeeding; RCT: Randomized controlled trial; SoC: Standard of care; UNICEF: United Nations International Children's Emergency Fund

\section{Acknowledgements}

We wish to acknowledge:

1. The Patrick J. McGovern Foundation for funding this research.

2. Erfan Mojaddam and Kim Walker for designing the mobile technology used to disseminate the intervention in this study.

3. The Digital MEdIC South Africa production team for their role in creating the intervention.

4. The Stanford Center for Health Education for supporting Digital MEdIC South Africa.

5. The Philani Mentor Mothers for their critical role in implementing this study on the ground.

6. Stellenbosch University for their role in supporting the early design phase of this study.

7. The ELMA Foundation for their generous support of our health education initiatives in South Africa.

8. The South African collaborating institutions and partners who co-created the 100\% Breastfed Intervention, including: UNICEF, The National Dept. of Health, the Western Cape Dept. of Health, The University of Cape Town, The University of Limpopo, Stellenbosch University, the University of Witwatersrand, the DG Murray Trust and the Philani Maternal Child Health and Nutrition Trust.

\section{Funding}

This study has been externally reviewed and funded following a competitive grant application and review process by the Patrick J. McGovern Foundation and by The Stanford Center for Health Education. The funders had no role in the design, data collection, analysis, interpretation of data or writing of the manuscript.

\section{Availability of data and materials \\ Not applicable.}

\section{Authors' contributions}

MA participated in all aspects of the study design and logistics and led the writing of the protocol. MT advised on both quantitative and qualitative design, oversaw the South African ethics approval process and edited the protocol paper. IL advised on the study design, set up the local study implementation systems and edited the protocol paper. AL advised on the quantitative study design, sample size calculation, local study implementation and edited the protocol paper. SM participated in the design and writing of the qualitative study components and edited the protocol paper. JJ advised on study design, oversaw the US ethics approval process and edited the protocol paper. AK set up the local study implementation systems, contributed to and edited the protocol paper. NM set up the local study implementation systems, contributed to and edited the protocol paper. SS set up the local study implementation systems, contributed to and edited the protocol paper. CP advised on all aspects of the study design, contributed to and edited the protocol paper.

TB supervised all aspects of the study design, the writing and editing of the protocol paper; he also conducted the power calculations and selected the statistical approaches for the quantitative analyses of effectiveness. All authors have read and approve of the final version of the manuscript.

\section{Ethics approval and consent to participate}

Ethical approval has been granted by the Stanford University IRB (Protocol \#46667) and the University of Stellenbosch IRB (Project ID \#6318 HREC/UREC Reference \#: N18/02/013), both recognized Ethical Review Committees. The investigators will consistently respect the principles of ethical research on human subjects, including respect for persons, beneficence and justice. Informed consent will be obtained in writing from all eligible participants before data collection begins and after the participants have been fully informed about the study.

\section{Consent for publication}

Not applicable. (This manuscript contains no identifiable data.)

\section{Competing interests}

The authors declare that they have no competing interests.

\section{Publisher's Note}

Springer Nature remains neutral with regard to jurisdictional claims in published maps and institutional affiliations.

\section{Author details \\ 'The Department of Pediatrics, Stanford University, Stanford, CA, USA. ${ }^{2}$ Stanford University's Digital Medical Education International Collaborative (Digital MEdIC), Cape Town, South Africa. ${ }^{3}$ Stanford Center for Health Education, Stanford, CA, USA. ${ }^{4}$ Institute for Life Course Health Research, Department of Global Health, Stellenbosch University, Stellenbosch, South Africa. ${ }^{5}$ Philani Maternal Child Health and Nutrition Trust, Cape Town, South Africa. ${ }^{6}$ Johns Hopkins Bloomberg School of Public Health, Baltimore, USA. ${ }^{7}$ Division of Epidemiology and Biostatistics, University of Cape Town School of Public Health, Cape Town, South Africa. ${ }^{8}$ Heidelberg Institute of Global Health (HIGH), Medical Faculty and University Hospital, Heidelberg University, Heidelberg, Germany. ${ }^{9}$ Stanford University, Stanford, CA, USA. ${ }^{10}$ Department of Global Health and Population, Harvard T.H Chan School of Public Health, Boston, USA. "1 Africa Health Research Institute (AHRI), Somkhele, KwaZulu-Natal, South Africa.}

Received: 14 December 2018 Accepted: 7 March 2019

Published online: 02 April 2019

\section{References}

1. World Health Organization (WHO). World health statistics 2016: monitoring health for the sustainable development goals. Geneva: WHO Report; 2016.

2. Liu L, Oza S, Hogan D, Chu Y, Perin J, Zhu J, et al. Global, regional, and national causes of under-5 mortality in 2000-15: an updated systematic analysis with implications for the sustainable development goals. Lancet. 2016;388(10063):3027-35.

3. Kuruvilla S, Bustreo F, Kuo T, Mishra CK, Taylor K, Fogstad H, Gupta GR, Gilmore K, Temmerman M, Thomas J, Rasanathan K. The Global strategy for women's, children's and adolescents' health (2016-2030): a roadmap based on evidence and country experience. Bull World Health Organ. 2016;1;94(5): 398.

4. You D, Hug L, Ejdemyr S, Idele P, Hogan D, Mathers C, et al. Global, regional, and national levels and trends in under-5 mortality between 1990 and 2015, with scenario-based projections to 2030: a systematic analysis by the UN inter-agency Group for Child Mortality Estimation. Lancet. 2015; 386(10010):2275-86

5. Victora CG, Bahl R, Barros AJ, França GV, Horton S, Krasevec J, et al. Breastfeeding in the 21st century: epidemiology, mechanisms, and lifelong effect. Lancet. 2016;387(10017):475-90.

6. Doherty T, Sanders D, Jackson D, Swanevelder S, Lombard C, Zembe W et al. Early cessation of breastfeeding amongst women in South Africa: an area needing urgent attention to improve child health. BMC Pediatr. 2012;12(1):105. 
7. National Department of Health (NDoH), Statistics South Africa (Stats SA), South African Medical Research Council (SAMRC), and ICF. South Africa Demographic and Health Survey 2016. Pretoria and Rockville: NDoH, Stats SA, SAMRC, and ICF; 2019.

8. Du Plessis L, Peer N, English R, Honikman S. Breastfeeding in South Africa: are we making progress? South African Health Review. 2016;2016(1):109-23.

9. Cohen RJ, Brown KH, Dewey K, Canahuati J, Rivera LL. Effects of age of introduction of complementary foods on infant breast milk intake, total energy intake, and growth: a randomised intervention study in Honduras. Lancet. 1994;344(8918):288-93.

10. Kimmons JE, Dewey KG, Haque E, Chakraborty J, Osendarp SJ, Brown KH. Low nutrient intakes among infants in rural Bangladesh are attributable to low intake and micronutrient density of complementary foods. J Nutr. 2005;135(3):444-51.

11. Haroon S, Das JK, Salam RA, Imdad A, Bhutta ZA. Breastfeeding promotion interventions and breastfeeding practices: a systematic review. BMC Public Health. 2013;13(3):S20.

12. Chapman DJ, Morel K, Anderson AK, Damio G, Pérez-Escamilla R. Breastfeeding peer counseling: from efficacy through scale-up. J Hum Lact. 2010;26(3):314-26.

13. Imdad A, Yakoob MY, Bhutta ZA. Effect of breastfeeding promotion interventions on breastfeeding rates, with special focus on developing countries. BMC Public Health. 2011;11(3):S24.

14. Lumbiganon P, Martis R, Laopaiboon M, Festin MR, Ho JJ, Hakimi M. Antenatal breastfeeding education for increasing breastfeeding duration. Cochrane Database Syst Rev. 2016(12):CD006425. https://doi.org/10.1002/ 14651858.

15. Slater MD. Entertainment education and the persuasive impact of narratives. Mahwah: Lawrence Erlbaum Associates Publishers; 2002.

16. Hinyard $L J$, Kreuter MW. Using narrative communication as a tool for health behavior change: a conceptual, theoretical, and empirical overview. Health Educ Behav. 2007;34(5):777-92

17. Rogers EM, Vaughan PW, Swalehe R, Rao N, Svenkerud P, Sood S. Effects of an entertainment-education radio soap opera on family planning behavior in Tanzania. Stud Fam Plan. 1999;30(3):193-211.

18. Shen F, Han J. Effectiveness of entertainment education in communicating health information: a systematic review. Asian J Commun. 2014:24(6):605-16.

19. Slater MD, Rouner D. Entertainment-education and elaboration likelihood: understanding the processing of narrative persuasion. Commun Theory. 2002;12(2):173-91.

20. Wilson KE, Beck VH. Entertainment outreach for women's health at CDC. J Womens Health Gend Based Med. 2002;11(7):575-8.

21. Aranda-Jan CB, Mohutsiwa-Dibe N, Loukanova S. Systematic review on what works, what does not work and why of implementation of mobile health (mHealth) projects in Africa. BMC Public Health. 2014;14(1):188.

22. Gurman TA, Rubin SE, Roess AA. Effectiveness of mHealth behavior change communication interventions in developing countries: a systematic review of the literature. J Health Commun. 2012;17(sup1):82-104.

23. Sondaal SFV, Browne $J$, Amoakoh-Coleman M, Borgstein A, Miltenburg AS, Verwijs $M$, et al. Assessing the effect of mHealth interventions in improving maternal and neonatal care in low-and middle-income countries: a systematic review. PLoS One. 2016:11(5):e0154664.

24. World Health Organization (WHO). mHealth: new horizons for health through mobile technologies: second global survey on eHealth. Geneva: WHO; 2011.

25. Labrique AB, Vasudevan L, Kochi E, Fabricant R, Mehl G. mHealth innovations as health system strengthening tools: 12 common applications and a visual framework. Glob Health: Sci Pract. 2013;1(2):160-71.

26. Källander K, Tibenderana JK, Akpogheneta OJ, Strachan DL, Hill Z, ten Asbroek AH, Conteh L, Kirkwood BR, Meek SR. Mobile health (mHealth) approaches and lessons for increased performance and retention of community health workers in low-and middle-income countries: a review. J Med Internet Res. 2013;15(1)

27. Diamond L. In search of democracy. Oxon: Routledge; 2015.

28. Kakihara M. Grasping a global view of smartphone diffusion: an analysis from a global smartphone study. 2014 International Conference on Mobile Business; 2014.

29. Kreutzer T. Generation mobile: online and digital media usage on mobile phones among low-income urban youth in South Africa. Cape Town: University of Cape Town; 2009
30. Barron P, Pillay Y, Fernandes A, Sebidi J, Allen R. The MomConnect mHealth initiative in South Africa: early impact on the supply side of $\mathrm{MCH}$ services. J Public Health Policy. 2016;37(2):201-12.

31. Seebregts C, Barron P, Tanna G, Benjamin P, Fogwill T. MomConnect: an exemplar implementation of the health normative standards framework in South Africa. South Afr Health Rev. 2016;2016(1):125-35.

32. Poushter J. Smartphone ownership and internet usage continues to climb in emerging economies. Pew Res Cent. 2016;22:1-44.

33. Shambare R. The adoption of WhatsApp: breaking the vicious cycle of technological poverty in South Africa. J Econ Behavior Stud. 2014;6(7):542.

34. Watkins JO, Goudge J, Gómez-Olivé FX, Griffiths F. Mobile phone use among patients and health workers to enhance primary healthcare: A qualitative study in rural South Africa. Soc Sci Med. 2018;198:139-47.

35. Coetzee B, Kohrman H, Tomlinson M, Mbewu N, Le Roux I, Adam M. Community health workers' experiences of using video teaching tools during home visits—a pilot study. Health Soc Care Community. 2018;26(2):167-75.

36. Rollins NC, Bhandari N, Hajeebhoy N, Horton S, Lutter CK, Martines JC, et al. Why invest, and what it will take to improve breastfeeding practices? Lancet. 2016;387(10017):491-504.

37. Tomlinson M, Rotheram-Borus MJ, Le Roux IM, Youssef M, Nelson SH, Scheffler A, et al. Thirty-six-month outcomes of a generalist paraprofessional perinatal home visiting intervention in South Africa on maternal health and child health and development. Prev Sci. 2016;17(8):937-48.

38. Black RE, Taylor CE, Arole S, Bang A, Bhutta ZA, Chowdhury AM, Kirkwood BR, Kureshy N, Lanata CF, Phillips JF, Taylor M. Comprehensive review of the evidence regarding the effectiveness of community-based primary health care in improving maternal, neonatal and child health: 8 . summary and recommendations of the Expert Panel. J Glob Health. 2017;7(1):010908. https://doi.org/10.7189/jogh.07.010908.

39. Mosavel M, Simon C, Van Stade D, Buchbinder M. Community-based participatory research (CBPR) in South Africa: engaging multiple constituents to shape the research question. Soc Sci Med. 2005;61(12):2577-87.

40. Bazzano AN, Martin J, Hicks E, Faughnan M, Murphy L. Human-centred design in global health: a scoping review of applications and contexts. PLoS One. 2017:12(11):e0186744.

41. Adam M, McMahon SA, Prober C, Bärnighausen T. Human-centered Design of Video-Based Health Education: an iterative, collaborative, communitybased approach. J Med Internet Res. 2019;21(1):e12128.

42. Brown T, Wyatt J. Design thinking for social innovation. Dev Outreach. 2010;12(1):29-43.

43. Groenewald P, Berteler M, Bradshaw D, Coetzee D, Cornelius K, Daniels J, et al. Western cape mortality profile 2010. Cape Town: South African Medical Research Council; 2013.

44. Goosen C, McLachlan M, Schübl C. Infant feeding practices during the first 6 months of life in a low-income area of the Western Cape Province. South Afr J Child Health. 2014:8(2):50-4.

45. le Roux IM, Tomlinson M, Harwood JM, O'Connor MJ, Worthman CM, Mbewu $\mathrm{N}$, et al. Outcomes of home visits for pregnant mothers and their infants: a cluster randomised controlled trial. AIDS. 2013;27(9):1461.

46. Rotheram-Borus MJ, Le Roux IM, Tomlinson M, Mbewu N, Comulada WS, Le Roux K, et al. Philani plus (+): a Mentor mother community health worker home visiting program to improve maternal and infants' outcomes. Prev Sci. 2011;12(4):372-88.

47. Hanington B, Martin B. Universal methods of design: 100 ways to research complex problems, develop innovative ideas, and design effective solutions. Beverly: Rockport Publishers; 2012.

48. Medlock MC, Wixon D, Terrano M, Romero R, Fulton B. Using the RITE method to improve products: A definition and a case study. Usability Professionals Association. 2002:51.

49. Burns C, Dishman E, Verplank W, Lassiter B. Actors, hairdos \& videotape-informance design. Boston: Conference Companion on Human Factors in Computing Systems; 1994.

50. Adam M. 100\% Breastfed Trailer 2018 [10.18.18]. Available from: https:// youtu.be/1sss8ViPKJo.

51. European Medicines Agency (EMA) Committee for Medicinal Products for Human Use. Guideline on adjustment for baseline covariates. London: EMA; 2014

52. Bai Y, Middlestadt SE, Peng C-YJ, Fly AD. Predictors of continuation of exclusive breastfeeding for the first six months of life. J Hum Lact. 2010;26(1):26-34. 
53. Barennes H, Empis G, Quang TD, Sengkhamyong K, Phasavath P, Harimanana A, et al. Breast-milk substitutes: a new old-threat for breastfeeding policy in developing countries. A case study in a traditionally high breastfeeding country. PLoS One. 2012;7(2):e30634.

54. Dennis CL. Breastfeeding initiation and duration: a 1990-2000 literature review. J Obstet Gynecol Neonatal Nurs. 2002;31(1):12-32.

55. Kitano N, Nomura K, Kido M, Murakami K, Ohkubo T, Ueno M, et al. Combined effects of maternal age and parity on successful initiation of exclusive breastfeeding. Prev Med Rep. 2016;3:121-6.

56. McCann MF, Liskin L, Piotrow PT, Rinehart W, Fox G. Breast-feeding fertility and family planning. Popul Rep J Fam Plan Programs. 1981;24:1-51.

57. Goga AE, Doherty T, Jackson DJ, Sanders D, Colvin M, Chopra M, et al. Infant feeding practices at routine PMTCT sites, South Africa: results of a prospective observational study amongst HIV exposed and unexposed infants-birth to 9 months. Int Breastfeed J. 2012;7(1):4.

58. Meedya S, Fahy K, Kable A. Factors that positively influence breastfeeding duration to 6 months: a literature review. Women Birth. 2010;23(4):135-45.

59. Mogre V, Dery M, Gaa PK. Knowledge, attitudes and determinants of exclusive breastfeeding practice among Ghanaian rural lactating mothers. Int Breastfeed J. 2016;11(1):12.

60. Hemming K, Girling A, Sitch A, Marsh J, Lilford R. Sample size calculations for cluster randomised controlled trials with a fixed number of clusters. BMC Medical Research Methodology. 2011;11:1.

61. Barnhart D, Hertzmark E, Liu E, Mungure E, Muya AN, Sando D, et al. Intracluster correlation estimates for HIV-related outcomes from care and treatment clinics in Dar Es Salaam, Tanzania. Contemp Clin Trials Commun. 2016:4:161-9.

62. Du Plessis LM. Commitment and capacity for the support of breastfeeding in South Africa: a paediatric food-based dietary guideline. South Afr J Clin Nutr. 2013;26(3):S120-S8.

63. Siziba L, Jerling J, Hanekom S, Wentzel-Viljoen E. Low rates of exclusive breastfeeding are still evident in four south African provinces. South Afr J Clin Nutr. 2015;28(4):170-9.

64. World Health Organization (WHO). Indicators for assessing infant and young child feeding practices. Part 1: definition. Geneva: WHO; 2008.

65. Greiner T. Exclusive breastfeeding: measurement and indicators. Int Breastfeed J. 2014;9(1):18.

66. Patton MQ. Qualitative evaluation and research methods. Thousand Oaks: SAGE Publications; 1990.

67. Bowen GA. Naturalistic inquiry and the saturation concept: a research note. Qual Res. 2008;8(1):137-52.

68. Tuckett A. Qualitative research sampling: the very real complexities. Nurse Res. 2004;12(1):47.

69. McMahon SA, Winch PJ. Systematic debriefing after qualitative encounters: an essential analysis step in applied qualitative research. BMJ Glob Health. 2018;3(5):e000837.

70. Creswell JW, Clark VLP. Designing and conducting mixed methods research. Thousand Oaks: SAGE publications; 2017.

71. Marcus AC, Crane LA. Telephone surveys in public health research. Med care. 1986;24(2):97-112.

72. Siemiatycki J. A comparison of mail, telephone, and home interview strategies for household health surveys. Am J Public Health. 1979;69(3):238-45.

73. Weeks MF, Kulka RA, Lessler JT, Whitmore RW. Personal versus telephone surveys for collecting household health data at the local level. Am J Public Health. 1983;73(12):1389-94.

74. Zou G. A modified poisson regression approach to prospective studies with binary data. Am J Epidemiol. 2004;159(7):702-6.

75. Charmaz K, Belgrave L. Qualitative interviewing and grounded theory analysis. The SAGE handbook of interview research: the complexity of the craft. Thousand Oaks: SAGE Publications; 2012.

76. Bazeley P, Jackson K. Qualitative data analysis with NVivo. Thousand Oaks: SAGE Publications; 2013

77. NVivo qualitative data analysis Software (edition 10). QSR International Pty Ltd; 2014.

78. Petty RE, Cacioppo JT. The elaboration likelihood model of persuasion. InCommunication and persuasion. New York: Springer; 1986. pp. 1-24

79. Moyer-Gusé E. Toward a theory of entertainment persuasion: explaining the persuasive effects of entertainment-education messages. Commun Theory. 2008;18(3):407-25.
80. Israel BA, Schulz AJ, Parker EA, Becker AB. Review of community-based research: assessing partnership approaches to improve public health. Annu Rev Public Health. 1998;19(1):173-202.

81. Viswanathan M, Ammerman A, Eng E, Garlehner G, Lohr KN, Griffith D, et al. Community-based participatory research: assessing the evidence. Evid Rep Technol Assess. 2004;(99):1-8.

82. Noel-Weiss J, Taljaard M, Kujawa-Myles S. Breastfeeding and lactation research: exploring a tool to measure infant feeding patterns. Int Breastfeed J. 2014;9(1):5.

83. Tylleskär T, Jackson D, Meda N, Engebretsen IMS, Chopra M, Diallo $\mathrm{AH}$, et al. Exclusive breastfeeding promotion by peer counsellors in sub-Saharan Africa (PROMISE-EBF): a cluster-randomised trial. Lancet. 2011;378(9789):420-7.

84. Centers for Disease Control (CDC). Infant Feeding Practices Study II (IFPS II). Atlanta: CDC; 2007.

85. LeFevre A. Impact evaluation of maternal messaging in India: study protocol. Baltimore: Johns Hopkins University; 2018.

86. Akinyinka MR, Olatona FA, Oluwole EO. Breastfeeding knowledge and practices among mothers of children under 2 years of age living in a military barrack in Southwest Nigeria. Int J MCH AIDS. 2016;5(1):1.

87. Mbada CE, Olowookere AE, Faronbi JO, Oyinlola-Aromolaran FC, Faremi FA, Ogundele $\mathrm{AO}$, et al. Knowledge, attitude and techniques of breastfeeding among Nigerian mothers from a semi-urban community. BMC Res Notes. 2013;6(1):552.
Ready to submit your research? Choose BMC and benefit from:

- fast, convenient online submission

- thorough peer review by experienced researchers in your field

- rapid publication on acceptance

- support for research data, including large and complex data types

- gold Open Access which fosters wider collaboration and increased citations

- maximum visibility for your research: over $100 \mathrm{M}$ website views per year

At BMC, research is always in progress.

Learn more biomedcentral.com/submissions 